\title{
Gemcitabine Re-challenge in Metastatic Soft Tissue Sarcomas: A Therapeutic Option for Selected Patients
}

\author{
ANA SEBIO ${ }^{1}$, ANASTASIA CONSTANTINIDOU ${ }^{1,2}$, CHARLOTTE BENSON $^{1}$, GEORGIOS ANTONIOU ${ }^{1}$, \\ CHRISTINA MESSIOU ${ }^{1,3}$, AISHA MIAH ${ }^{1}$, SHANE ZAIDI ${ }^{1}$, ANN PETRUCKEVITCH $^{4}$, \\ OMAR AL-MUDERIS ${ }^{1}$, KHIN THWAY ${ }^{1,3}$, WINETTE T. VAN DER GRAAF ${ }^{1,3}$ and ROBIN L. JONES ${ }^{1,3}$ \\ ${ }^{1}$ Sarcoma Unit, Royal Marsden Hospital, London, U.K.; \\ ${ }^{2}$ Medical School, University of Cyprus, Nicosia, Cyprus; \\ ${ }^{3}$ Institute of Cancer Research, London, U.K.; \\ ${ }^{4}$ Statistics Department, Royal Marsden Hospital, London, U.K.
}

\begin{abstract}
Background/Aim: Treatment options for patients with metastatic soft tissue sarcomas are limited. Rechallenge with a previously successful gemcitabine-based regimen is common. There are no published data to support this practice. Patients and Methods: We conducted a retrospective search to identify patients re-challenged with gemcitabine-based chemotherapy (GBC) from 2003 to 2015. Results: Twenty-nine patients re-challenged with gemcitabine were identified. The response rate for initial GBC was 55\% $(n=15)$ and for re-challenge $G B C 26 \%(n=6)$. The median progression-free survival was 11.1 months (95\% CI=7.2-11.9) for initial $G B C$ and 5.3 months (95\%CI=2.0-7.5) for rechallenge GBC. Overall survival following gemcitabine rechallenge was 12.2 months (95\%CI=7.0-18.2). Twelve out of 26 evaluable patients (46\%) treated with re-challenge $G B C$ experienced grade 3-4 adverse events (CTCAE 4.03) with $31 \%(n=8)$ of patients requiring dose reduction. Conclusion: In selected patients, gemcitabine re-challenge can be considered in advanced sarcomas, however, this approach is associated with toxicity.
\end{abstract}

Soft tissue sarcomas (STS) are rare tumours that represent approximately $1 \%$ of adult cancers (1). STS comprise more than 60 different entities of which leiomyosarcoma (LMS), liposarcoma (LPS) and undifferentiated pleomorphic sarcoma are among the most common subtypes (2). For localized

This article is freely accessible online.

Correspondence to: Robin L. Jones, Sarcoma Unit, Royal Marsden Hospital, Fulham Road, SW3 6JJ, London, U.K. Tel: +44 207808 2590, Fax: +44 207808 2113, e-mail: robin.jones4@ nhs.net

Key Words: Soft tissue sarcomas, chemotherapy, gemcitabine, rechallenge. disease, surgery with or without neoadjuvant/adjuvant radiation is the mainstay of management $(3,4)$. The role of neoadjuvant/adjuvant chemotherapy is controversial, but it may be considered in patients with high risk of relapse $(5,6)$. Despite advances in therapy, approximately $50 \%$ of patients will develop recurrent/metastatic disease. The cornerstone of treatment for metastatic/locally advanced inoperable disease consists of palliative systemic chemotherapy.

Anthracycline-based therapy (either alone or in combination) is the standard first-line therapy $(7,8)$. Over the last few years, a number of new agents have been approved for $2 \mathrm{nd}$ and subsequent line therapy, including trabectedin, pazopanib and eribulin $(9,10)$. However, older chemotherapy drugs such as ifosfamide and gemcitabine still belong to the standard armamentarium for advanced disease.

Gemcitabine is a pyrimidine antimetabolite drug frequently used in combination with docetaxel. In a phase II trial conducted by the Sarcoma Alliance for Research through Collaboration (SARC) in unselected metastatic STS, the combination of gemcitabine plus docetaxel was superior to gemcitabine alone in terms of response rate $(16 \% \mathrm{vs.} 8 \%)$, median progression-free (6.2 vs. 3.0 months) and median overall survival (17.9 vs. 11.5 months) at the expense of a higher toxicity profile (11). The French phase II TAXOGEM trial, evaluated gemcitabine $v s$. gemcitabine docetaxel as 2nd-line therapy in patients with metastatic leiomyosarcoma. The endpoint of the study was response rate, which was $19 \%$ for gemcitabine alone and $24 \%$ for the combination arm (not statistically significant). The median progression-free survival was 6.3 months for non-uterine leiomyosarcomas treated with the combination compared to 3.8 months for the gemcitabine monotherapy arm (12).

Gemcitabine can also be combined with dacarbazine. In a phase II trial conducted by the Spanish Sarcoma Group (GEIS), gemcitabine plus dacarbazine was compared to dacarbazine alone in previously treated STS patients. The 
combination arm yielded a higher response rate $(12 \% \mathrm{vs}$. $4 \%$ ), as well as longer progression-free (4.2 vs. 2 months; $\mathrm{HR}=0.58 ; p=0.005)$ and overall survival (16.8 vs. 8.2 months; HR=0.55; $p=0.014)(13)$.

Despite the recent advances and the incorporation of new drugs, treatment options for metastatic STS remain limited. Consequently, maximising the existing regimens by rechallenging selected patients who relapse after a reasonable interval with the same effective chemotherapy agent can be considered. Re-challenge with doxorubicin is not possible due to the risk of cardiotoxicity. In a retrospective study, ifosfamide re-challenge was found to be a viable option for metastatic STS, specifically synovial sarcoma (14).

Gemcitabine-based re-challenge may also be an option in selected patients previously benefitting from gemcitabine. To our knowledge, there are no published data supporting this strategy and therefore, the aim of this study was to evaluate the efficacy and toxicity of gemcitabine-based re-challenge in STS patients treated at a single referral centre.

\section{Materials and Methods}

Institutional approval was obtained prior to commencing the study (Royal Marsden Clinical Audit Committee approval 7/11/2017). The prospectively maintained Royal Marsden Sarcoma Unit database was retrospectively reviewed to identify patients re-challenged with gemcitabine between 2003 and 2015. Gemcitabine-based re-challenge was defined as treatment with gemcitabine as mono-therapy or in combination with another chemotherapy agent (docetaxel or dacarbazine) in any line and subsequent re-treatment with gemcitabine in a further line. Initial gemcitabine-based chemotherapy was defined as the chemotherapy line that gemcitabine was first used.

Clinical data and demographics as well as treatment details were obtained from the electronic patient records. This study was not limited to any specific histology and in all cases the histologic diagnosis was confirmed by a specialist STS pathologist (KT).

Chemotherapy schedules, response and toxicity assessment Gemcitabine was administered as monotherapy at $1,200 \mathrm{mg} / \mathrm{m}^{2}$ Days 1 and 8 every 3 weeks or in combination with docetaxel (gemcitabine $900 \mathrm{mg} / \mathrm{m}^{2}$ or $675 \mathrm{mg} / \mathrm{m}^{2}$ Days 1 and 8 and docetaxel $75 \mathrm{mg} / \mathrm{m}^{2}$ Day 8) or dacarbazine (gemcitabine $1,800 \mathrm{mg} / \mathrm{m} 2$ Days 1 and 15 and dacarbazine $500 \mathrm{mg} / \mathrm{m}^{2}$ Days 1 and 15). Re-challenge gemcitabine-based regimes could be the same as the first or differ at the discretion of the treating oncologist.

Response assessment was performed using CT scans. We retrospectively reviewed CT scans performed every 2 to 3 cycles using Response Evaluation Criteria in Solid Tumors (RECIST 1.1). Rereview of radiologic response was only possible for imaging performed from 2006 onwards (following installation of electronic archiving).

Cancer Institute Common Terminology Criteria for Adverse Events (CTCAE) were used to record grade 3 and 4 toxicities.

Statistical analysis. Clinical activity to gemcitabine-based chemotherapy (GBC) was measured following RECIST 1.1 as the percentage of patients achieving a complete response (CR) or a partial response (PR).
Table I. Baseline characteristics.

\begin{tabular}{lc}
\hline & Overall patients (N=29) \\
& $\mathrm{N}(\%)$ \\
\hline Age & \\
Median (range) & $48(30-60)$ \\
Gender & \\
Male & $8(28)$ \\
Female & $21(72)$ \\
Primary site location & \\
Uterus & $16(55)$ \\
Retroperitoneum & $4(14)$ \\
Extremities & $4(14)$ \\
Other & $5(17)$ \\
Histology & \\
Leiomyosarcoma & $25(86)$ \\
Angiosarcoma & $1(3.5)$ \\
Spindle cell sarcoma & $1(3.5)$ \\
Endometrial Stromal Sarcoma & $1(3.5)$ \\
Kaposi Sarcoma & $1(3.5)$ \\
Grade & \\
Low & $2(7)$ \\
Intermediate & $7(24)$ \\
High & $14(48)$ \\
Unknown & $6(21)$ \\
First GBC regimen & \\
Gemcitabine-docetaxel & $25(86)$ \\
Gemcitabine monotherapy & $4(14)$ \\
Re-challenge GBC regimen & \\
Gemcitabine-docetaxel & $19(66)$ \\
Gemcitabine monotherapy & $9(31)$ \\
Gemcitabine-DTIC & $1(3)$ \\
\hline & \\
\hline & \\
& \\
&
\end{tabular}

GBC: Gemcitabine-based chemotherapy; DTIC: dacarbacine.

Progression-free survival (PFS) for first GBC treatment was calculated from the first treatment date until disease progression and for second GBC from the first treatment date until disease progression, death by any cause or last follow-up.

Overall survival of the series was calculated from the original diagnosis date until death by any cause or last follow up. Overall survival (OS) after GBC re-challenge was calculated from the first treatment date of the second GBC treatment to the date of death by any cause or last follow-up. For OS and PFS analysis, survival curves and survival medians were estimated at $95 \%$ confidence using the Kaplan-Meier estimator. A 95\% level of significance was used for all the statistical tests. All statistical analyses were done using the statistical software STATA version 13.1.

\section{Results}

Baseline characteristics. From 2003 to 2015 a total of 29 patients were re-challenged with gemcitabine-based chemotherapy (GBC). The median age at diagnosis was 48 years and $72 \%$ of the patients were female. The majority of patients were diagnosed with metastatic leiomyosarcoma $(n=25$, $86 \%)$ mostly uterine leiomyosarcoma $(n=16,55 \%)$ and the 

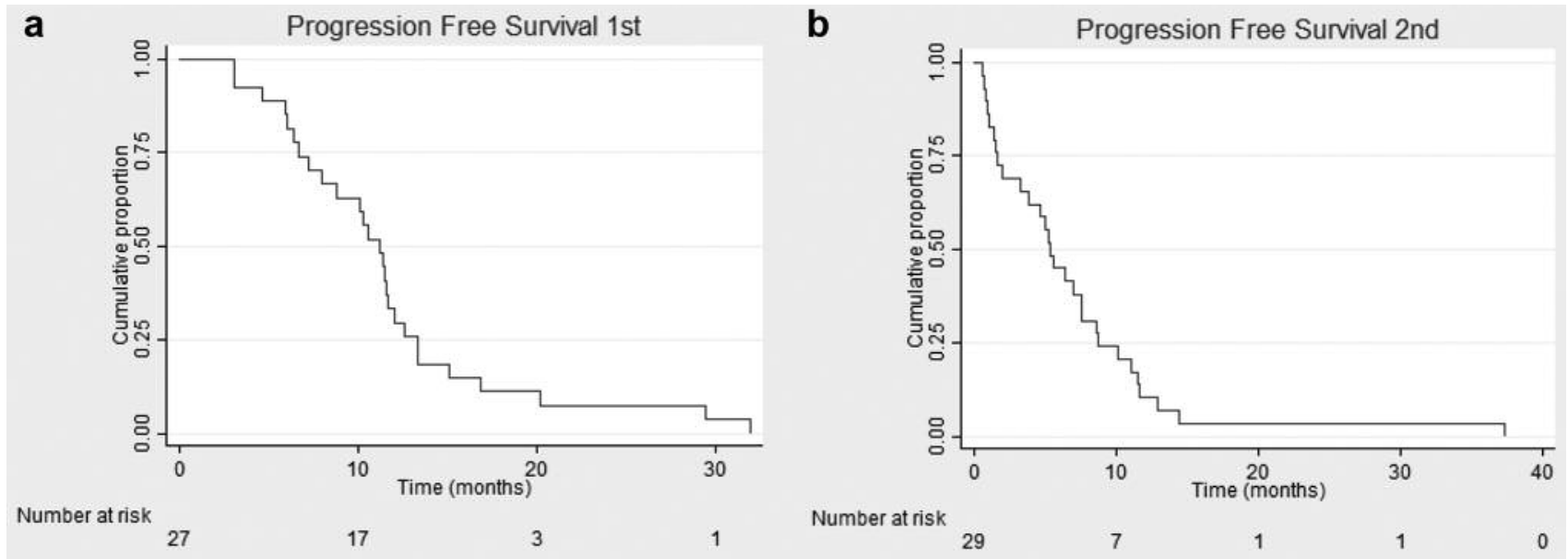

Figure 1. Progression-free survival following initial and re-challenge gemcitabine-based chemotherapy. a) Progression-free survival following initial gemcitabine-based chemotherapy. b) Progression-free survival following re-challenge gemcitabine-based chemotherapy.

majority of the tumours were intermediate to high grade $(n=21)$. The baseline characteristics of this series are shown in Table I.

\section{Response and toxicity}

Initial gemcitabine-based chemotherapy. Thirty-eight percent $(\mathrm{n}=11)$ of patients received initial GBC as 1st-line therapy, $45 \%(n=13)$ as $2 n d-$ line and $17 \%(n=5)$ as 3rd-line or greater. Twenty-five $(86 \%)$ patients received gemcitabine plus docetaxel as initial GBC and the remainder gemcitabine alone as initial GBC. The median number of cycles administered was 5.4. Twenty-eight patients were evaluable for response, as one patient did not have RECIST measurable disease. The response rate was $55 \%(n=15)$.

Nine $(31 \%)$ of the evaluable patients experienced grade 34 toxicity and $7(24 \%)$ required a dose reduction.

Re-challenge gemcitabine-based chemotherapy. Re-challenge GBC consisted of gemcitabine-docetaxel in $66 \%(n=19)$, gemcitabine mono-therapy in $31 \%(n=9)$ and gemcitabinedacarbazine in $3 \%(n=1)$ of patients. Re-challenge GBC was administered as 2nd-line therapy in $21 \%(\mathrm{n}=6)$, as 3rd-line in $34 \%(n=10)$ and 4 th-line/greater in $45 \%(n=13)$ of patients. The median number of cycles administered was 4.2. Twenty-three patients were evaluable for response and the response rate for gemcitabine re-challenge was $26 \%(n=6)$.

Twelve out of 26 patients $(46 \%)$ treated with re-challenge GBC experienced grade 3-4 adverse events and 31\% $(n=8)$ required a dose reduction.

Only patients with leiomyosarcoma had RECIST radiological responses $(n=15 / 25)$ to either initial or rechallenge GBC. No radiological responses were observed in the 4 other histological subtypes. A total of 5 patients had a response to initial GBC and also to gemcitabine re-challenge.
Progression-free and overall survival. The median PFS was 11.1 months $(95 \% \mathrm{CI}=7.2-11.9)$ for initial GBC. For rechallenge with gemcitabine-based chemotherapy median PFS was 5.3 months $(95 \% \mathrm{CI}=2.0-7.5)$ (Figure 1).

Following initial GBC, the majority of patients $(n=23)$ had a PFS of more than 6 months. Two of the patients not achieving 6 months PFS had angiosarcoma and spindle cell sarcoma.

Following re-challenge GBC, a total of 16 patients had a PFS longer than 4 months.

The median overall survival from sarcoma diagnosis of this series was 50.6 months $(95 \% \mathrm{CI}=33.4-69.9)$. The median overall survival for patients treated with gemcitabine rechallenge was 12.2 months (95\% CI=7.0-18.2) (Figure 1).

\section{Discussion}

Our retrospective study suggests that re-challenge with a previously successful gemcitabine-based regimen can be a therapeutic option for selected metastatic soft tissue sarcoma patients.

It is important to highlight that in our clinical practice we selected patients achieving a good and prolonged response with initial GBC before considering re-challenge with gemcitabine. Furthermore, we would not employ rechallenge GBC in those with progressive disease to initial GBC and those with a poor performance status.

In our study, the majority of patients had leiomyosarcoma (mostly grade 2 and 3 ) highlighting the higher sensitivity of this subtype to gemcitabine-based chemotherapy (15).

The majority of patients were treated with gemcitabine plus docetaxel as initial (55\%) and re-challenge (25\%) GBC.

The median PFS for initial GBC was 11.1 months, which is longer than that reported in prospective trials. This is 
probably due to the inherent selection bias of this series (i.e. to be considered for re-challenge, patients had durable benefit to initial GBC). In the GeDDis trial, comparing 1stline gemcitabine-docetaxel versus doxorubicin, the median PFS for gemcitabine-docetaxel was 6 months. The median PFS for re-challenge GBC was relatively long (5.3 months), considering many patients received re-challenge GBC as 4thline or greater. Moreover, the median overall survival following re-challenge $\mathrm{GBC}$ was 12.2 months, but again this emphasises the selection of this chemo-sensitive cohort.

Gemcitabine plus docetaxel is associated with considerable toxicity. In the phase II trial published in 2002, the haematological grade 3-4 toxicities were $21 \%$ neutropenia (neutropenic fever 6\%), 29\% thrombocytopenia and $15 \%$ anaemia, and non-haematological grade 3-4 toxicities included $21 \%$ dyspnoea or $21 \%$ fatigue (16). In the phase III GeDDis trial, 285 serious adverse events were reported of which 111 (39\%) were febrile neutropenia, fever and neutropenia, which led to $18 \%$ dose reductions (8). Other gemcitabine combinations or gemcitabine monotherapy have been associated with a lower percentage of severe adverse events $(12,13)$, but in our study fewer patients were treated with these regimens. Grade 3-4 toxicities in the re-challenge GBC setting were high $(46 \%)$ and a considerable number of patients needed a dose reduction $(31 \%)$. The fact that patients were treated in the 4th-line or greater may have contributed to this toxicity profile (i.e. heavily pre-treated). Therefore, when considering gemcitabine re-challenge the risk of increased toxicity should be discussed carefully with patients. If toxicity is a potential concern, then gemcitabine mono-therapy could be used.

The limitations of our study include its retrospective nature, the small number of patients, the lack of radiological review for some patients as well as the lack of patient reported outcome measures (which are particularly relevant in the setting of pre-treated advanced cancer).

\section{Conclusion}

The outcome of patients with metastatic soft tissue sarcoma remains poor with few systemic therapy options. Gemcitabine re-challenge could be a potential option for selected patients. Based on our results, patients with leiomyosarcoma achieving a PFS longer than 6 months following initial GBC could benefit the most. However, it is important to discuss the potential for toxicity carefully with patients, particularly if rechallenge is offered to heavily pre-treated patients.

\section{Acknowledgements}

During this work, Ana Sebio was a recipient of a Juan Rodés contract (JR14/00006) and a mobility grant (M-AES, 2017) from Instituto Salud Carlos III.
The Authors acknowledge support from the NIHR Royal Marsden/ICR Biomedical Research Centre.

\section{References}

1 Fletcher D, Bridge $\mathrm{J}$ and Hogendoorn PC: Who classification of tumours of soft tissues and bone, 4th ed. Lyon: Iarc press, 2013.

2 Corey RM, Swett K and Ward WG: Epidemiology and survivorship of soft tissue sarcomas in adults: A national cancer database report. Cancer Med 3(5): 1404-1415, 2014.

3 Clark MA, Fisher C, Judson I and Thomas JM: Soft-tissue sarcomas in adults. N Engl J Med 353(7): 701-711, 2005.

4 O'Sullivan B, Davis AM, Turcotte R, Bell R, Catton C, Chabot P, Wunder J, Kandel R, Goddard K, Sadura A, Pater J and Zee B: Preoperative versus postoperative radiotherapy in soft-tissue sarcoma of the limbs: A randomised trial. Lancet 359(9325): 2235-2241, 2002.

5 Pervaiz N, Colterjohn N, Farrokhyar F, Tozer R, Figueredo A and Ghert M: A systematic meta-analysis of randomized controlled trials of adjuvant chemotherapy for localized resectable soft-tissue sarcoma. Cancer 113(3): 573-581, 2008.

6 Gronchi A, Ferrari S, Quagliuolo V, Broto JM, Pousa AL, Grignani G, Basso U, Blay JY, Tendero O, Beveridge RD, Ferraresi V, Lugowska I, Merlo DF, Fontana V, Marchesi E, Donati DM, Palassini E, Palmerini E, De Sanctis R, Morosi C, Stacchiotti S, Bague S, Coindre JM, Dei Tos AP, Picci P, Bruzzi P and Casali PG: Histotype-tailored neoadjuvant chemotherapy versus standard chemotherapy in patients with high-risk soft-tissue sarcomas (isgsts 1001): An international, open-label, randomised, controlled, phase 3, multicentre trial. Lancet Oncol 18(6): 812-822, 2017.

7 Judson I, Verweij J, Gelderblom H, Hartmann JT, Schoffski P, Blay JY, Kerst JM, Sufliarsky J, Whelan J, Hohenberger P, Krarup-Hansen A, Alcindor T, Marreaud S, Litiere S, Hermans C, Fisher C, Hogendoorn PC, dei Tos AP, van der Graaf WT, European O, Treatment of Cancer Soft T and Bone Sarcoma G: Doxorubicin alone versus intensified doxorubicin plus ifosfamide for first-line treatment of advanced or metastatic softtissue sarcoma: A randomised controlled phase 3 trial. Lancet Oncol 15(4): 415-423, 2014.

8 Seddon B, Strauss SJ, Whelan J, Leahy M, Woll PJ, Cowie F, Rothermundt C, Wood Z, Benson C, Ali N, Marples M, Veal GJ, Jamieson D, Kuver K, Tirabosco R, Forsyth S, Nash S, Dehbi HM and Beare S: Gemcitabine and docetaxel versus doxorubicin as first-line treatment in previously untreated advanced unresectable or metastatic soft-tissue sarcomas (geddis): A randomised controlled phase 3 trial. Lancet Oncol 18(10): 1397-1410, 2017.

9 Demetri GD, Schoffski P, Grignani G, Blay JY, Maki RG, Van Tine BA, Alcindor T, Jones RL, D'Adamo DR, Guo M and Chawla S: Activity of eribulin in patients with advanced liposarcoma demonstrated in a subgroup analysis from a randomized phase iii study of eribulin versus dacarbazine. J Clin Oncol 35(30): 3433-3439, 2017.

10 van der Graaf WT, Blay JY, Chawla SP, Kim DW, Bui-Nguyen B, Casali PG, Schoffski P, Aglietta M, Staddon AP, Beppu Y, Le Cesne A, Gelderblom H, Judson IR, Araki N, Ouali M, Marreaud S, Hodge R, Dewji MR, Coens C, Demetri GD, Fletcher CD, Dei Tos AP, Hohenberger P, Tissue ES, Bone Sarcoma G and group Ps: Pazopanib for metastatic soft-tissue sarcoma (palette): A randomised, double-blind, placebocontrolled phase 3 trial. Lancet 379(9829): 1879-1886, 2012. 
11 Maki RG, Wathen JK, Patel SR, Priebat DA, Okuno SH, Samuels B, Fanucchi M, Harmon DC, Schuetze SM, Reinke D, Thall PF, Benjamin RS, Baker LH and Hensley ML: Randomized phase ii study of gemcitabine and docetaxel compared with gemcitabine alone in patients with metastatic soft tissue sarcomas: Results of sarcoma alliance for research through collaboration study 002 [corrected]. J Clin Oncol 25(19): 2755-2763, 2007.

12 Pautier P, Floquet A, Penel N, Piperno-Neumann S, Isambert N, Rey A, Bompas E, Cioffi A, Delcambre C, Cupissol D, Collin F, Blay JY, Jimenez M and Duffaud F: Randomized multicenter and stratified phase ii study of gemcitabine alone versus gemcitabine and docetaxel in patients with metastatic or relapsed leiomyosarcomas: A federation nationale des centres de lutte contre le cancer (fnclcc) french sarcoma group study (taxogem study). Oncologist 17(9): 1213-1220, 2012.

13 García-del-Muro X, López-Pousa A, Maurel J, Martín J, Martínez-Trufero J, Casado A, Gómez-España A, Fra J, Cruz J, Poveda A, Meana A, Pericay C, Cubedo R, Rubió J, Juan AD, Laínez N, Carrasco JA, Andrés Rd and Buesa JM: Randomized phase ii study comparing gemcitabine plus dacarbazine versus dacarbazine alone in patients with previously treated soft tissue sarcoma: A spanish group for research on sarcomas study. Journal of Clinical Oncology 29(18): 2528-2533, 2011.
14 Noujaim J, Constantinidou A, Messiou C, Thway K, Miah A, Benson C, Judson I and Jones RL: Successful ifosfamide rechallenge in soft-tissue sarcoma. Am J Clin Oncol 41: 147$151,2015$.

15 Bathan AJ, Constantinidou A, Pollack SM and Jones RL: Diagnosis, prognosis, and management of leiomyosarcoma: Recognition of anatomic variants. Curr Opin Oncol 25(4): 384389, 2013.

16 Hensley ML, Maki R, Venkatraman E, Geller G, Lovegren M, Aghajanian C, Sabbatini P, Tong W, Barakat R and Spriggs DR: Gemcitabine and docetaxel in patients with unresectable leiomyosarcoma: Results of a phase ii trial. J Clin Oncol 20(12): 2824-2831, 2002.
Received November 25, 2018

Revised December 6, 2018

Accepted December 7, 2018 